\title{
LA INTERDEPENDENCIA EMPRESARIAL FACTOR DE CALIDAD EN LA GESTIÓN - CASO SECTOR EMPRESARIAL PESQUERO PERUANO
}

\author{
Mildred Paredes Tarazona
}

\begin{abstract}
RESUMEN
Se estudia la generación de relaciones de interdependencia centrada en factores psicosociales: Motivación, Percepción e Intereses que propicien negocios compartidos en una muestra representativa del sector empresarial exportador pesquero peruano. El análisis de datos con estadísticos no paramétricos señalan que las variables estudiadas no son muy significativas para conformar alianzas estratégicas en la perspectiva del desarrollo sostenible.
\end{abstract}

Palabras Clave: Motivación de Logro, Motivación de Poder, Motivación de Autorrealización, Intereses, Percepción a negocios compartidos, alianzas estratégicas, interdependencia.

\begin{abstract}
The generation of interdependence relationships is studied centered in factors psychosocials: Motivation, Perception and Interests that propitiate business shared in a representative sample of the sector managerial exporter fishing Peruvian. The analysis of data with statistical non pamétricos points out that the studied variables are not very significant to conform strategic alliances in the perspective of the sustainable development.
\end{abstract}

Key Words: Motivation of Achievement, Motivation of Power, Motivation of Autorrealización, Interests, Perception to shared business, strategic alliances, interdependence. 


\section{REFERENCIAS TEÓRICAS}

Los diferentes enfoques y conceptos sobre los efectos de la globalización en nuestros países y la búsqueda de soluciones en términos de interdependencias empresariales. Para tal fin se entiende por interdependencia como la capacidad de respuesta hacia las exigencias del medio o contexto comercial, productivo o de servicios de dos o más personas, o grupos u organizaciones sean empresariales o de carácter institucional, a través de la formación de alianzas estratégicas, cuyo objetivo primordial es ser más competitivos.

Mintzberg \& Quinn (1993: 11-15), afirman que la estrategia es una posición, un medio para ubicar una organización en su medio ambiente, por tanto, la estrategia viene a ser la fuerza mediadora. Una forma particular de establecer una estrategia es la mencionada por Astley y Fombrum (1983: 7-19), quienes introducen la noción de estrategia colectiva. Es aquella que busca promover la cooperación entre organizaciones y aún entre posibles competidores (en biología equivale a los animales que para protegerse se agrupan); para Philip Selznick (1957: 18) la estrategia es una perspectiva compartida por y entre los miembros de una organización, a través de sus intenciones y sus acciones y sin desvirtuarla, y para Michael Porter, (1995: 31-34), ésta debe tener el carácter de "estrategia competitiva", la cual consiste en desarrollar una amplia fórmula de cómo la empresa va a competir, cuáles deben ser sus objetivos y qué políticas serán necesarias para alcanzar tales objetivos.

Quinn y Mintzberg (1993: 846) señalan el aspecto innovativo de las fusiones y alianzas; como su variedad cada vez mayor de las relaciones creativas donde participan las grandes empresas: negocios conjuntos, consorcios, sociedades limitadas, garantes de mercados primarios, fondos para patrocinar actividades académicas, capitalistas dispuestos al riesgo, accionistas externos y demás. Estas formas rivalizan con la amplia gama de financiamiento, inventiva y estructuras de redes que vienen generado los empresarios individuales.

Para ello la empresa moderna indica Andrés Navarro (IPAE 1996: 78), las alianzas son parte medular de su estrategia, en la medida que expresan la capacidad de trabajar con otros que a veces tienen intereses distintos, incluso, contrapuestos, además de la capacidad de concertar con competidores, consumidores y proveedores.

O sea, la interdependencia en organizaciones es altamente favorable para ayudar a la eficiencia del líder en el entendimiento de la influencia de su poder y efectividad (Tjosvold 1986), al que Gundlach y Cadotte (1994) señalan que las firmas llegan a la interdependencia como un resultado atractivo en el cambio económico, hacia el control de recursos necesarios para sus objetivos.

Para Pfeffer y Salancik el control externo de las organizaciones tiene su origen en la interdependencia que, al mismo tiempo, era corolario de la naturaleza de las organizaciones como sistemas abiertos (Jeffrey Pfeffer 1992,213); Michel \& Hambrick (1992: 9-37), argumentan que las alianzas son parte de una estrategia de diversificación, tal es el caso de las empresas integradas verticalmente, demandan la cohesión y el conocimiento básico en los equipos de alta gerencia.

La interdependencia corporativa, es el grado por la cual la performance de una corporación como un todo, depende del compartir los recursos y coordinación dentro de los que conforman sus unidades o socios, donde la interacción este encaminada a un desarrollo sustentable, fortalecida por la motivación de logro, la que generará una interdependencia de resultados es decir «las consecuencias o resultados logrados por un actor social son interdependientes con los de otro actor social» (Pfeffer y Salansik,1978, Tedeschi, Schlenker y Bonome 1973). Así, el grado de interdependencia afecta motivaciones, comportamientos y percepciones y éstas a la formación de interdependencia. 
De Santamaría (Mankeliunas 1991), indica que una de las áreas más estudiadas de la motivación humana es la necesidad de logro. Los primeros intentos de medir la motivación en forma sistemática y repetible se deben a McClelland, Atkinson, Clark y Lowell (1953). De estos estudios surgieron gran cantidad de estudios teóricos y experimentales. Concluyendo en llamar «motivación de logro» a la disposición, relativamente estable, de buscar éxito o logro (Atkinson: 1966); Así como la tendencia a alcanzar el éxito en situaciones que implican la evaluación del desempeño de una persona, en relación con estándares de excelencia (McClelland: 1953). En el contexto de la teoría del aprendizaje, se ha definido la motivación de logro como un sistema de autorrefuerzo en el que los estándares de excelencia se consideran reglas de contingencia para la autoevaluación que sigue al éxito y al fracaso (Kuhl: 1978).

Este motivo "realización" ha sido estudiado en detalle por McClelland y sus colaboradores, durante más de veinte años se han acumulado sobre ella datos de investigación, en laboratorio, sobre terreno, en la historia y en muchos países distintos. Los datos refuerzan considerablemente la hipótesis de que la realización, como motivo, se desarrolla en culturas y en familias en las que se hace hincapié en la evolución independiente del individuo. En contraste, la realización como motivación de poca intensidad aparece asociada a familias en las que el niño es más de pendiente de sus padres y de importancia menor a la de ellos.

Madsen (1967:256) reformula una hipótesis secundaria: «El motivo realización está determinado por expectativas continuamente crecientes relativas a las posibilidades y demandas de realización» y McClelland concluye que, los niveles altos de necesidad de logro de la elite empresarial de una sociedad reflejan un mayor desarrollo económico, independientemente de las condiciones sociales y políticas de la sociedad, así, McClelland logra demostrar en sus investigaciones que este tipo de motivación encamina al desarrollo económico.

Hacia 1965 Kock, en un estudio mucho más extensivo, muestra también el efecto de una alta expectativa de éxito sobre la expansión de los negocios. Las calificaciones de los gerentes de firmas finlandesas generalmente pequeñas, se encuentran asociadas significativamente con varias medidas de expansión mercantil, en especial con los incrementos del número de trabajadores, en el valor bruto de la producción, en la rotación y en la inversión bruta a lo largo de un período de ocho años. Wainer y Rubin hacia 1967, dieron cuenta de una correlación similar entre las calificaciones de éxito de los empresarios y la expansión de sus firmas de investigación y desarrollo en los Estados Unidos.

Es también de fundamental importancia y transcendencia que uno de los criterios de calificación de la orientación al éxito comprende la competencia con otros, aunque enfatiza McClelland (1970: 103-104), que un interés primario está dado por el hecho que la competencia se constituye en una norma de excelencia; la que consiste en hacer las cosas mejor de lo que las hemos hecho antes, bien en función de alguna norma objetiva o la norma de conducta de alguna otra persona.

De ahí que las expectativas involucren también estándares de excelencia con respecto a tales tareas. Todo lo que sabemos acerca de la motivación al éxito revela que una persona que la tenga alta no se restringe a los medios actualmente disponibles de alcanzar sus metas, sino que persigue medios nuevos y alternativos. La competencia percibida exclusivamente en función de la lucha por recursos limitados es una imagen de poder.

En esta perspectiva debemos tomar en consideración el enfoque de la motivación por la personalidad formulaba por Argyris (Luthans \& Kreitner 1989), quien señala que la 
personalidad de todo líder, como es el del empresario, en tanto cumpla su rol debe estar dirigida a crear las condiciones para que su compañero o socio pase de una posición dependiente a una independiente. Esta postura de la personalidad está relacionada con la necesidad de autorrealización, es decir el deseo de llegar a ser cada vez más lo que es. Esto nos lleva a pensar y constatar en el contexto actual, que la necesidad de éxito ha dejado de ser una motivación puramente individual, dando paso a una responsabilidad compartida en los negocios contemporáneos.

La percepción se constituye de esta forma en la evaluación de la conducta de los demás y de los propios estados o expectativas, así como, el reconocimiento de las emociones, la percepción que el individuo tiene de su medio físico y social y la formación de impresiones y procesos de atribución. (Santoro 1979: 114). Por ello «percibir» no es recibir pasivamente estimulación; es seleccionar, formular hipótesis, decidir y procesar la estimulación, eliminando, aumentando o disminuyendo aspectos de la estimulación. Debido al contexto en el cual se realiza el proceso de percepción, por las características permanentes o estables del medio físico, social y cultural, y por el tipo de relaciones que el individuo establece con dicho medio, éste es simultáneamente afectado y transformado en función del desarrollo cultural y social, sobre la base de programas y planes que suponen preconcepciones y expectativas sobre la realidad. Este tipo de percepción es conocido también como percepción social o cognición social.

Como señalan Montero y colaboradores (1986: 167) desempeñamos un papel activo en la creación de nuestras experiencias, que conjuntamente con e! lenguaje y e! bagaje cultural se logra categorizar los eventos de manera diferente. Este proceso nos ayuda a estructurar nuestro mundo personal, e! que difiere según la experiencia, propósitos o las relaciones que entre éstas pudieran establecerse.

Por otra parte, nuestro mundo de las experiencias está dotado de cierta estabilidad, siendo el acto perceptual aquél que genera un entendimiento que podemos utilizar como base para la acción. El proceso perceptual es un logro de la persona y la percepción no existiría sin una activa solución de problemas, puesto que el aprendizaje tiene influencia muy significativa en la percepción pero dentro de marco de actividad orientada hacia objetivos. Es decir, nuestras percepciones tienen significado y sentido que se derivan de nuestras experiencias pasadas y de nuestras metas actuales. Sin la presencia de! sentido y el significado como agentes activos organizadores, la percepción no existiría tal como la conocemos.

Gladys Montesinos (1986: 158-59), refiere de otro lado que, cuando interactuamos con personas podemos darnos cuenta de que ellas ajustan su comportamiento en respuesta al nuestro. Podemos tratar de cambiar el comportamiento de la otra persona pero ella también puede tener propósitos y objetivos. Así, cuando tratamos de entender el comportamiento de la otra persona, debemos determinar cómo entiende ella nuestro propio comportamiento, en términos estrictamente connotativo o con significado actitudinal. En una interacción cada persona debe entender no solamente lo que la otra persona está haciendo, sino también lo que él o ella ven como estímulos relevantes en e! ambiente. De ahí que, la percepción de las personas y de su comportamiento es estructurada, estable y dotada de sentido, J. C. Rivera (1996: 22-28), enfatiza que la percepción social consiste en la anticipación de hechos sobre la base de motivaciones y especialmente, a las expectativas que tiene e! sujeto respecto al entorno.

Son Wolitzky y Wachtel (Wolman, 1980: 361), quienes confirman que los estudios de la personalidad trajeron consigo e! reconocimiento de la importancia de la motivación en 
todos los aspectos de la conducta. El hecho de que las necesidades influyan en la percepción ha constituido desde hace tiempo un hecho asumido. No obstante, debemos reconocer que la naturaleza de esta influencia es extraordinariamente compleja.

Desde una perspectiva humanística, Bugental enfatizó que e! hombre proyecta por medio de la realización de propósitos, de las valoraciones, de la creatividad y del reconocimiento de las significaciones. La intencionalidad humana es la base sobre la que e! hombre construye su identidad, lo cual le distingue de otras especies. Sabe que sus elecciones establecen diferencias, que no es un espectador sino un participante en sus experiencias. (Wolman, 1980: 345-346).

En conclusión, la percepción como proceso que nos posibilita tomar conocimiento de la realidad, es llevado a cabo por los perceptores (quienes toman conocimiento de o ve a... ) y de los percibidos (quienes toma conocimiento o el que es visto por ... ). Estos perceptores, pueden ser organizaciones empresariales, de diverso tamaño y complejidad, pues son sistemas sociales, regidos por interacciones entre ellas, que a su vez son calificadas por las características estructurales y culturales de las maneras aceptadas de "hacer las cosas" de la empresa.

En cuanto a los efectos en el contexto, Katona (1965) se refiere principalmente a los papeles sociales: "El individuo tiene un papel y una función diferente, según e! grupo al que pertenece. No debe esperarse que el individuo se comportará de la misma manera en diferentes situaciones de grupo". Las preferencias individuales sobre las cuales se basan las tendencias económicas no cambian frecuentemente y de súbito; están formadas por cambios en el medio de las personas, en su situación y en sus oportunidades, así como por tendencias en sus predisposiciones psicológicas.

Vicuña (1991) define el interés, como el motivo o meta que impulsa a la acción selectiva, cuya temporalidad puede ser inmediata y mediata o a largo plazo. Es un componente básico de la motivación, que indica lo qué se está buscando. Por ejemplo, mejorar la calidad del producto. Aclarando, se puede decir que la motivación se resuelve en el interés, según Tolman (1987: 118), la expectativa como "disposición medios-fin", la motivación es importante como determinante del desempeño, pues está dirigida o guiada con un propósito por los determinantes cognitivos.

La clave de! crecimiento económico es el hombre y sus valores y actitudes. Estos tienen que cambiarse para acelerar el proceso de crecimiento; siendo la educación, los medios de comunicación masivos, la ideología, el liderazgo participativo-democrático o el súper liderazgo, las variables que producen o generan dicha modernización. En esta dinámica, el conocimiento es útil de algún modo al hombre y a la empresa o institución, para moldear su destino. Tal es el caso de que un empresario no puede convertirse en próspero industrial hasta que haya aprendido a valorar la inversión a largo plazo o hasta que se haya adiestrado en las técnicas modernas de administración empresarial.

Así, Cárdenas (1996: 4) señala que para la empresa contemporánea, uno de los indicadores fundamentales del entorno son las grandes tendencias que engloban a las sociedades, más aún si se trata de una economía globalizada. Estas tendencias son producto complejo del quehacer de millones de empresarios, trabajadores y funcionarios de las diversas naciones y países. Estas tendencias denominadas mega tendencias por Naisbitt (1996, IPAE).

Estas mega tendencias, al lado del problema más grave de esta época; la pobreza, pueden actuar con una fuerza insospechada para impedir las acciones regionales, internacionales o nacionales dirigidas a promover el desarrollo sostenible. En este marco de aspiraciones de 
desarrollo sostenible y tendencias que de algún modo pueden atentar contra tales propósitos, corresponde también un papel a las empresas latinoamericanas, en general a las empresas del mundo en desarrollo, las que deberán desarrollarse bajo los conceptos de una nueva cultura que involucre objetivos no sólo económicos, sino también de naturaleza humana, social y medioambiental.

\subsection{FORMULACION DEL PROBLEMA}

Las empresas pesqueras peruanas se desenvuelven en diferentes tipos de actividades en la producción, comercio y servicios de los productos pesqueros. En este sector, se observan diversos problemas derivados de la no aparente predisposición a participar o integrar conglomerados de negocios conjuntos, o relaciones productivas y comerciales de triangulación, utilización de franquicias u otras modalidades empresariales que en el contexto actual se vienen llevando a cabo con gran aceleración en el marco del proceso de globalización.

Por otro lado, es un hecho preocupante dada la importancia pesquera del Perú en el contexto latinoamericano y mundial, que según las estadísticas oficiales del año 1995 publicadas por el Ministerio de Pesquería y OLDEPESCA, en el país se realizaron aproximadamente 8'994,700 TM., con volúmenes de exportación de productos pesqueros que ascendieron en dicho año a 2.0 millones de TM., por un valor de 875.2 millones de dólares. Al comparar estas cifras con las logradas por otros países de la región, se observa que contrasta por el significativo nivel de volúmenes exportados con bajos valores de exportación. A manera de ilustración se cita los casos de Chile y México. Para el caso del primer país, éste registra 1.7 millones de TM. de exportación con valores de 1,507 millones de dólares en el mismo año (1995) y, el sector empresarial pesquero de México capturó en el mismo año 1 '404,384 TM. con volúmenes de exportación de 190,400 TM. ascendiendo su valor a 680 millones de dólares.

Se reconoce asimismo, altos niveles de explotación y la alta calidad y diversidad del recurso pesquero nacional, con valores que no justifican los índices mencionados, alcanzados por el Sector Empresarial Pesquero. Sector que cuenta con una población aproximada de 245 empresas dedicadas a la producción y comercio de harina y aceite de pescado, conservas y congelados, entre los más significativos productos de exportación. De la base estimada de empresas posibilita indicar a efectos de tomar en cuenta de que, entre ellas o con sus similares de la región $u$ otros mercados inicien un flujo de relaciones interdependientes para conformar bloques empresariales de ofertas sostenibles de productos con niveles de producción y calidad optimizada, mediante las diferentes modalidades de negocios internacionales, como las triangulaciones comerciales, modernas $\mathrm{u}$ otras modalidades de negocios compartidos en el marco de la globalización económica, para acceder y satisfacer a los mercados internacionales como al nacional. Esta tendencia es generada por las señales del mercado así como por la decisión de los empresarios hacia las modalidades de los negocios compartidos.

En el plano sectorial de la pesca se observa que para hacer frente a los problemas que plantea el contexto de una economía global izada, la actividad pesquera peruana requiere optar modalidades de negocios compartidos que consignen y promuevan los intereses de la producción y el comercio de los productos pesqueros del país, mediante la concertación de acciones conjuntas para la consolidación y ampliación de sus mercados de exportación e interno; la cooperación científica y tecnológica; y su desarrollo empresarial. Para responder 
al acelerado proceso de cambio que se verifica en el comercio internacional y a sus efectos en los esquemas de integración de países y empresas, que buscan proteger y ampliar sus mercados, así como complementar sus factores de producción y elevar sus índices de productividad y competitividad, a fin de lograr el desarrollo económico sostenible que en su naturaleza trae el desarrollo humano.

De ahí que para tener un conocimiento global de esta problemática se ha tomado a una muestra representativa del sector empresarial pesquero peruano para responder las siguientes interrogantes:

1. Poseen los empresarios pesqueros las motivaciones e intereses que propician la conformación de bloques o negocios conjuntos de Empresas Pesqueras que permitan obtener mayores ventajas en el comercio internacional?

2. Cuáles son las percepciones de los empresarios peruanos hacia la conformación de negocios compartidos para el comercio internacional?

3. Hay interés de los empresarios peruanos para facilitar la promoción y la participación de las empresas pesqueras peruanas en la conformación de organizaciones internacionales?

\section{3 OBJETIVOS}

1. Identificar las motivaciones, e intereses y percepciones que facilitan la relación de interdependencia entre empresarios pesqueros hacia la formación de bloques $o$ modalidades asociativas empresariales para una gestión de calidad.

2. Proponer criterios para el desarrollo de políticas de gestión para la competitividad del sector empresarial pesquero peruano.

3. Determinar las percepciones empresariales que coadyuvan la gestión de formas asociativas según sus intereses hacia el Comercio Internacional.

\subsection{LIMITACIONES DE LA INVESTIGACION}

La investigación se centra en la interdependencia por el mercado de productos pesqueros en un contexto en el que hay poca disposición de los inversionistas y/o gerentes del sector para responder a la encuesta.

\subsection{VARIABLES}

Variables Independientes: Motivaciones, Percepciones e Intereses.

Variables Intervinientes: Producto Pesquero, Volumen de Exportación, Valores de Exportación y Mercado.

Variable Dependiente: Interdependencia Empresarial.

\section{METODOLOGÍA}

\section{Población}

Productores de harina y aceite de pescado, constituido aproximadamente por 245 empresas en varias modalidades empresariales tales como: consorcios, otras empresas de gestión individual (OLDEPESCA 95).

En el siguiente cuadro se presenta la distribución de estas empresas. 


\section{Cuadro $\mathbf{N}^{\circ} 1$ \\ Número de empresas por plantas y capacidad instalada para el procesamiento de pescado y productos pesqueros 1995}

\begin{tabular}{|c|c|c|c|c|c|c|c|c|}
\hline \multirow[t]{2}{*}{ Departamento } & \multicolumn{2}{|c|}{ ENLATADO } & \multicolumn{2}{|l|}{ HARINA } & \multicolumn{2}{|c|}{ CONGELADO } & \multicolumn{2}{|c|}{ CURADO } \\
\hline & $\begin{array}{c}\mathbf{N}^{0} \text { de } \\
\text { plantas }\end{array}$ & $\begin{array}{c}\text { Capac. } \\
\text { Instal. } \\
\text { CJS/TUR } \\
\text { (8H) }\end{array}$ & $\begin{array}{c}\mathbf{N}^{\circ} \text { de } \\
\text { plantas }\end{array}$ & $\begin{array}{c}\text { Capac. } \\
\text { Instal. } \\
\text { CJS/TUR } \\
(8 H)\end{array}$ & $\begin{array}{c}\mathbf{N}^{\circ} \text { de } \\
\text { plantas }\end{array}$ & $\begin{array}{c}\text { Capac. } \\
\text { Instal. } \\
\text { CJS/TUR } \\
(8 H)\end{array}$ & $\begin{array}{c}\mathbf{N}^{\circ} \text { de } \\
\text { plantas }\end{array}$ & $\begin{array}{c}\text { Capac. } \\
\text { Instal. } \\
\text { CJS/TUR } \\
(\mathbf{8 H})\end{array}$ \\
\hline Tumbes & 0 & 0 & 1 & 9 & 9 & 183.58 & 2 & 84 \\
\hline Piura & 11 & 20357 & 12 & 299 & 17 & 715.1 & 5 & 239 \\
\hline La Libertad & 3 & 4665 & 1 & 18 & 2 & 33.6 & 0 & 0 \\
\hline Ancash & 40 & 118866 & 38 & 1405 & 5 & 244.2 & 5 & 256.6 \\
\hline Lima & 39 & 29885 & 18 & 344 & 8 & 109.5 & 3 & 321.7 \\
\hline Ica & 8 & 9644 & 9 & 235 & 13 & 168.65 & 3 & 198.5 \\
\hline Junín & 0 & 0 & 0 & 0 & 1 & 2 & 0 & 0 \\
\hline Arequipa & 7 & 4267 & 5 & 120 & 3 & 19.4 & 0 & 0 \\
\hline Moquegua & 1 & 4630 & 5 & 320 & 2 & 19.6 & 1 & 17 \\
\hline Tacna & 3 & 1396 & 0 & 0 & 2 & 10 & 0 & 0 \\
\hline Total & 112 & 193710 & 89 & 2750 & 62 & 1505.63 & 19 & 116.8 \\
\hline
\end{tabular}

Fuente: Dirección General D.G.T. MPE.

29/10/91 Elaborado: OLDEPESCA

Nota: Para fines de la toma de muestra estadística se considera 71 empresas al considerarse a PESCA PERU como una sola unidad con 20 plantas procesadoras y Sindicato Pesquero del Perú con 8 plantas.

\section{MUESTRA Y BASES DE LA ESTRATIFICACIÓN}

Está subdividida en estratos que podrían aproximarse en términos estadísticos como mutuamente excluyentes por el Producto que emplean diversas especies (88) pero que juntos incluyen los componentes significativos de la población del sector productivo pesquero. Por otra parte este diseño conduce a estimados muestra les que tienen una mayor con fiabilidad que la obtenible de otra manera, como el muestreo por azar simple. De ahí que el muestreo por distribución óptima posibilite un mayor control por el empleo de la ubicación geográfica, que nos lleva a determinar por adelantado, el número de empresas e instituciones de los que se sacará la muestra de cada uno de los cuatro productos determinados, y planear el trabajo. El muestreo fue estratificado, las características usadas para subdividir el universo en los estratos fueron los siguientes:

1. Productos: a )Conservas, b )Congelados, c)Harina y d)Aceite de Pescado, e)Secos y t)Salados.

2. Distribución Geográfica, Volumen de Producción y Valores de las Exportaciones, de tal forma que permita definir la proporcionalidad relativa de las Plantas Pesqueras.

\section{TAMAÑO DE LOS ESTRATOS}

Se estima la muestra a partir de la media de las empresas pesqueras peruanas según el giro de productos señalados. Trabajado al $10 \%$ del margen de error con el 0.05 nivel de confianza. Por lo tanto se requiere un total de 26 empresas cuya distribución por fijación porcentual equivalente a la distribución poblacional es como sigue: 


\begin{tabular}{|l|c|c|c|c|c|}
\hline Empresas & $\begin{array}{c}\text { Tam. } \\
\text { N }\end{array}$ & $\begin{array}{c}\text { Pobl } \\
\text { \% }\end{array}$ & $\begin{array}{c}\text { Tam. } \\
\text { N }\end{array}$ & $\begin{array}{c}\text { Mues. } \\
\text { \% }\end{array}$ & $\begin{array}{c}\text { Aplic. } \\
\text { N }\end{array}$ \\
\hline Harina & 71 & 29 & 9 & 29 & 9 \\
Conservas & 112 & 44 & 12 & 49 & 13 \\
Congelado & 92 & $2 \mathrm{~S}$ & 7 & 25 & 15 \\
\hline TOTAL & 245 & 100 & 26 & 100 & 37 \\
\hline
\end{tabular}

Se distribuyeron 89 encuestas de acuerdo al directorio de ADEX, La Sociedad Nacional de Pesquería y de las empresas ranqueadas en TOP Perú 5000 del año 1995, lográndose 37 (coincidente con registro de 36 de CONASEV) respuestas anónimas de empresarios e inversionistas del sector, .

\section{DISEÑO DE LA INVESTIGACIÓN}

Se ha empleado como primer paso en el proceso de investigación concluyente, un DISEÑO DE INVESTIGACION DESCRIPTIVO - COMPARATIVO.

\section{TÉCNICA DE RECOLECCIÓN DE DATOS}

Se midieron los indicadores inferidas del comportamiento, a través de la encuesta diseñada exprofesamente, aplicada a los 37 empresarios pesqueros peruanos de la muestra de empresas.

La encuesta tiene el Formato de Cuestionario elaborado con los principios y normas de construcción de la Escala de Actitudes. La validez y confiabilidad del contenido se estableció por el criterio de jueces (expertos y ejecutivos en pesquería, administración, economía, psicología y metodología).

La escala de actitudes, según señala Fred Kerlinger (1988) es el instrumento de medición, constituido por un conjunto de símbolos o valores numéricos, de tal manera que se puedan asignar por regla a los representantes legales de las empresas o su conducta. A quienes se aplica la escala, indicándose la asignación de valoración en grados por la posesión de los empresarios o directivos en cualquier aspecto que la escala mida. El diseño de la Escala está fundamentada en los principios teóricos y metodológicos señalados por Osgood (1976), Thurstone y Likert.

Los aspectos que mide la encuesta son precisados en áreas, dimensiones e indicadores. Totalizan 67 preguntas o reactivos diseñados en positivo y en tercera persona en referencia al contexto latinoamericano, formuladas tomando en cuenta la población de la muestra encuestada, representantes legales de las empresas, sean inversionistas, gerentes 0 directivos de las empresas pesqueras con la más alta decisión.

Por las características que proporciona la situación interpersonal de la entrevista, (no estructurada o no estandarizada) especialmente por ser una técnica que posibilita obtener información o datos que complementen al análisis cualitativo, para el caso, el lenguaje propio en la formulación de las preguntas o reactivos, para evitar suspicacias o reacciones adversas que suscitan toda evaluación.

\section{TÉCNICAS DE ANÁLISIS}

Para la aceptación o rechazo de las hipótesis se emplea el método de la Estadística Inferencial al 0.05 de margen de error.

Para el tratamiento descriptivo se ha empleado el JI cuadrado $\left(\mathrm{X}^{2}= \pm>3.84\right)$ y para establecer las diferencias según el giro de producto pesquero se ha empleado el Análisis de la Razón Critica Porcentual (RC\%).Asimismo se empleó el análisis T (Diferencia de Promedios).

Para determinar la interdependencia se utiliza el análisis de la correlación de Pearson. 


\section{RESULTADOS Y ANALISIS DE LAS MOTIVACIONES, INTERESES Y PERCEPCIONES DE LOS EMPRESARIOS PESQUEROS PERUANOS.}

\section{DESCRIPCIÓN DE LAS MOTIVAOONES, PERCEPCIONES E INTERESES}

Los datos del cuestionario tabulados para cada uno de los indicadores respecto a: motivación del logro, motivación de poder y motivación de autorrealización; percepción a los problemas empresariales, modalidad de negocios compartidos. Finalmente los intereses por la pesca sostenible, gestión, mercado de los productos pesqueros que propicien la conformación de alianzas estratégicas y otras formas de gestión de negocios compartidos de las empresas pesqueras.

Luego los porcentajes obtenidos de los indicadores señalados se analizarán intra e intergrupal.

El primer análisis con el propósito de identificar las Motivaciones, Intereses y Percepciones que predominan significativamente en el grupo general y dentro de cada giro de producción en particular.

Con el segundo análisis la comparación intergrupal se efectúa con el propósito de tipificar si fuere el caso los componentes psicosociales que son propios del giro de producción.

Resultados que a continuación se presentan:

\section{Motivación de Logro}

El análisis endogrupal de la muestra total examinada para el caso de motivación de logro presenta, un promedio de 5.14 que comparándola con la media escalar normativa es igual a 5 , encontramos que el nivel motivacional del logro se encuentra dentro de los límites del promedio, muy concentrados alrededor de la media normativa, tal como puede verse en el Cuadro $\mathrm{N}^{\circ} 02$.

\section{Cuadro $\mathbf{N}^{\circ} 3$}

Media aritméticas y de dispersión calculada y teórica en función a la motivación de logro

\begin{tabular}{|l|c|}
\hline ESTADIGRAFO & VALOR \\
\hline Media Escalar & 5.00 \\
Desviación Escalar & 3.16 \\
Media Calculada & 5.14 \\
Desviación Escalar & 1.06 \\
\hline
\end{tabular}

A continuación se describen los diferentes indicadores de la escala de la motivación de logro en el Cuadro $\mathrm{N}^{\circ} 3$, los mismos se explican según los resultados:

1. Indicadores significativos en los empresarios en su conjunto según el análisis con el JI cuadrado $\left(x^{2}=>+3.84\right)$ :

a) Para invertir en la innovación tecnológica. (86\% - Item 2).

b) Para implementar programas de capacitación del recurso humano para el trabajo pesquero.(87\% - Item 3).

c) Prioritariamente las empresas latinoamericanas necesitan modernizar tecnológicamente sus procesos productivos para elaborar sus productos pesqueros.(81\% - Item 4).

d) Prioritariamente las empresas latinoamericanas necesitan estandarizar según las normas técnicas internacionales sus productos pesqueros. (84\% - Item 5).

e) Están fuertemente de acuerdo que la empresa quiere de sus ejecutivos que se logren un posicionamiento en el mercado.(95\% - Item 8).

f) Están totalmente de acuerdo que los ejecutivos de empresas pesqueras de América deben 
capitalizar los fracasos para mejorar la gestión empresarial. (89\%-Item 10).

g) Asimismo consideran que los ejecutivos de empresas pesqueras de América Latina que deben correr riesgos y enfrentar retos empresariales manejables (65\%-Item 9)

2.Está significativamente ausente $\left(x^{2}>3.84\right)$ en el grupo de empresarios la motivación para establecer relaciones conjuntas entre empresas para mejorar transacciones comerciales. (Item 1)

3.Tendencias Espúreas: Distribución porcentual casi equivalente en pro y en contra $\left(x^{2}=< \pm 3.84\right)$ son:

a) Promocionarse el producto pesquero en ferias y exposiciones (Item 6).

b) Concertar y establecer relaciones conjuntas con otras empresas por línea de producción, (Item 7).

Descripción de Motivación Logro Endogrupal - Por Giro de Producto

Los empresarios de las Empresas Conserveras, presentan los Indicadores Significativos para establecer relaciones conjuntas entre empresas para mejorar transacciones comerciales.

Y, como tendencias espúreas:

a) Que el producto pesquero elaborado por las empresas latinoamericanas necesita promocionarse en ferias y exposiciones.

b) Que la gestión de las empresas pesqueras de Latinoamérica necesita concertar y establecer relaciones conjuntas con otras empresas por línea de producción.

Entre los Indicadores significativamente ausentes $\left(x^{2}=+>-3.84\right)$ en el grupo empresarial de Harina de Pescado están:

a) No están dispuestos a un corto plazo para establecer relaciones conjuntas entre empresas para mejorar transacciones comerciales.

b) No consideran que el producto pesquero elaborado por las empresas latinoamericanas necesita prioritariamente promocionarse en ferias y exposiciones.

Y, como tendencia espuria jos ejecutivos de empresas pesqueras de América Latina deben correr riesgos y enfrentar retos empresariales manejables.

El Indicador Ausente significativo del grupo del Empresas de Congelados es la disposición a corto plazo para establecer relaciones conjuntas entre empresas para mejorar transacciones comerciales.

\section{MOTIVACION DE PODER}

La motivación de Poder en la muestra examinada, en el análisis endogrupal, los empresa nos peruanos pesqueros presentan, un promedio de 4.35 que comparada con la media escalar normativa es igual a 3 , en este caso es un punto mayor al promedio escalar, no obstante la concentración del grupo cae dentro del área del promedio, razón por la cual podemos afirmar que la Motivación de Poder se encuentra en un nivel medio, tal como se puede ver en el Cuadro No.4. 


\section{Cuadro No. 4}

\section{Medias aritméticas y de dispersión calculada y teórica en función a la motivación de poder}

\begin{tabular}{|l|c|}
\hline ESTADIGRAFO & VALOR \\
\hline Media Escalar & 3.00 \\
Desviación Escalar & 2.00 \\
Media Calculada & 4.35 \\
Desviación Escalar & 1.18 \\
\hline
\end{tabular}

Con respecto a los 6 indicadores significativos de la escala de la Motivación del Poder, los empresarios peruanos pesqueros presentan los siguientes resultados:

a) Hay disposición del $81 \%$, del empresario pesquero peruano para participar de una red internacional de información (Item 2)

b) El $81 \%$ tiene disposición para propiciar la consolidación y ampliación de sus mercados de exportación. (Item 3).

c) Asimismo el $84 \%$ de los empresarios tienen disposición para que, la gestión de las empresas pesqueras de Latinoamérica mantengan la oferta exportable competitiva del producto pesquero (Item 4)

d) El empresariado de América Latina no tiene disposición para asociarse para diversificar su empresa (78\%-Item 1)

e) Están fuertemente de acuerdo que la empresa pesquera requiere de sus ejecutivos, que se tenga control de la información comercial interregional (84\% Item 5)

f) Así como concuerda en un $84 \%$ en que se requiere la influencia o control de los medios de apoyo a las exportaciones. (Item 6)

Motivación de Poder por Giro de Producto Pesquero

Los empresarios de las empresas conserveras presentan los siguientes los componentes significativamente ausentes según el análisis de $\mathrm{x}^{2}>=-3.84$ :

a) El empresariado de América Latina no tiene disposición a corto plazo para asociarse para diversificar su empresa.

b) La disposición del empresariado pesquero de América Latina no está orientada a propiciar la consolidación y ampliación de sus mercados de exportación.

El Empresariado de Harina de Pescado presenta los componentes significativamente ausentes según el análisis de $\mathrm{x}^{2}>=-3.84$ :

a) No tiene disposición a corto plazo para asociarse para diversificar su empresa.

b) La disposición del empresariado no está orientada a propiciar la consolidación y ampliación de sus mercados de exportación.

Y, como Tendencia Espúrea: para incrementar su participación en una red internacional de información

Los componentes significativamente ausentes en el grupo de empresarios en el rubro de congelados,

a) No tiene disposición a corto plazo para asociarse para diversificar su empresa.

b) No están de acuerdo con la disposición a propiciar la consolidación y ampliación de sus mercados a exportación. 


\section{LA MOTIVACION DE AUTORREALIZACION}

El análisis endogrupal de la motivación de autorrealización ayudará a precisar la presencia de la motivación de logro como la de poder de los empresarios pesqueros peruanos que están dirigidos al desarrollo de la empresa y coadyuvar al desarrollo sostenido del país.

En el análisis, encontramos una media calculada de 6,51 siendo la media escalar normativa de 4,5. En este caso el valor promedio calculado, cae por encima de la media desviación estándar escalar respecto a su media, lo que significa que la motivación de autorrealización presenta una clara presencia hacia la autorrealización; tal como puede verse en el siguiente Cuadro No.6.

\section{Cuadro $\mathbf{N}^{\circ} 6$ \\ Medias Aritméticas y de Dispersión calculada y teórica en función a la motivación de autorrealización}

\begin{tabular}{|l|c|}
\hline ESTADIGRAFO & VALOR \\
\hline Media Escalar & 4.50 \\
Desviación Escalar & 2.87 \\
Media Calculada & 6.51 \\
Desviación Escalar & 1.37 \\
\hline
\end{tabular}

\section{DESCRIPCIÓN INTERGRUPAL}

El análisis de la razón crítica porcentual $(\mathrm{RC} \%)$ que a continuación se detalla en según el tipo de motivación.

La comparación de los componentes de la motivación de logro por el tipo de producto pesquero mediante el análisis de la razón critica, presenta los siguientes resultados observados:

1. Los empresarios de Harina de Pescado y los de Conservas tienen la misma motivación de logro.

2. Tampoco difieren en la motivación de logro, los empresarios de Conservas con los de Congelados.

3. Hay diferencia significativa entre los empresarios de Harina de Pescado y los de

Congelados en los ítems 6 (RC\% 2,07), es decir es mayor el componente de la motivación de logro en los empresarios de Congelados, referente a que éstos consideran necesario que el producto pesquero elaborado por las empresas latinoamericanas, se promocione en ferias y exposiciones.

Luego de la Comparación Intergrupal de la Motivación de Poder, entre los empresarios pesqueros según el giro de producto, se dan los siguientes resultados, mediante el análisis de la Razón Critica Porcentual (RS.):

1. Entre los empresarios de Conservas y de Harina de Pescado no se dan diferencias significativas, en la comparación entre los componentes de la motivación de poder.

2. Entre los empresarios de Conservas y de Congelados tampoco se presentan diferencias significativas proporcionada por el estadístico RC\%.

3. Hay diferencia significativa (RC\% 2.079) en los empresarios de Harina, quienes presentan mayor disposición para incrementar la participación en una red internacional de información, que en los empresarios de congelados. 


\section{PERCEPCION DE LOS EMPRESARIOS PESQUEROS}

Se identifican los problemas considerados prioritarios por los empresarios pesqueros, y como evaluar las típicas Organizaciones internacionales en el comercio internacional.

Los empresarios pesqueros de los tres giros de productos coinciden que los cinco principales problemas para la empresa son los siguientes según el orden de prioridad:

Cuadro No. 11

Percepción de los empresarios pesqueros peruanos a los problemas de la empresa

\begin{tabular}{|l|c|c|c|}
\hline \multicolumn{1}{|c|}{ EMPRESAS PESQUERAS } & Promedi & Interpret. & DS \\
\hline Total Promedio de Acceso a financiamiento & 2,1 & $1^{\circ}$ & 1,1643 \\
Total Promedio de Altos Costos bienes y & $2 ; 2$. & $2^{\circ}$ & 1,0168 \\
Total Promedio de Insuficiente tamaño mercado & 3,5 & $4^{\circ}$ & 1,3314 \\
Total Promedio de Recursos Humanos No espec & $3 ; 2$. & $3^{\circ}$ & $1 ; 2.178$ \\
Total Promedio de Incipiente Investigación & 4,0 & $5^{\circ}$ & 1,3707 \\
\hline
\end{tabular}

Descripción de la percepción al tipo de modalidad de negocios internacionales compartidas.

En la percepción a las modalidades de negocios empresariales internacionales compartidos se utiliza una escala con ítems dicotomizados de tres indicadores, donde la percepción favorable es igual a 3 y la percepción desfavorable igual a $\mathrm{O}$. La media aritmética encontrada en el estudio es de 1.32 que cae por debajo de la media aritmética teórica menos media desviación estándar, lo que indica que la percepción de los empresarios pesqueros peruanos a estos tipos de modalidad de negocios empresariales tiende a ser desfavorable, presentados en el Cuadro No.12.

\section{Cuadro $\mathbf{N}^{\circ} 12$}

Medias aritméticas y de dispersión calculada y teórica en función a la percepción a modalidades de negocios internacionales

\begin{tabular}{|l|c|}
\hline ESTADIGRAFO & VALOR \\
\hline Media Escalar & 1.50 \\
Desviación Escalar & 1.11 \\
Media Calculada & 1.32 \\
Desviación Escalar & 0.56 \\
\hline
\end{tabular}

A continuación se describe los diferentes indicadores de la escala de percepción a las modalidades de negocios internacionales compartidos procesados mediante el análisis con el J1 cuadrado (x2>3.84)

1. Percepción significativa $\left(x^{2}=5.76\right)$, positivamente a formar joint-venture entre latinoamericanos (Item 1).

2. Percepción significativa negativa $\left(x^{2}=-27.04\right)$ a adquirir franquicias y otras formas (Item 2).

3. Percepción a las modalidades de negocios conjuntos, tanto a favorecer o desaprobar, no está definida $\left(\mathrm{x}^{2}=-0.64\right)$ a constituir Tradings Co. (Item 3).

\section{La Percepción de los Empresarios por Giro de Producto a la Modalidad de Negocios} Internacionales Compartidas.

El grupo de empresarios de conservas presentan la percepción significativamente negativa a adquirir franquicias y otras formas $\left(\mathrm{x}^{2}=-49\right)$. 
Teniendo una percepción no definida a formar joint-venture entre latinoamericanos $\left(x^{2}=0.64\right)$ y a constituir trading $\left(x^{2}=-0.64\right)$.

Por parte del grupo de empresarios de Harina presentan significativa mente una percepción negativa evidente a adquirir franquicias y otras formas $\left(x^{2}=-100\right)$ y a constituir tradings $\left(x^{2}=-11.56\right)$.

Los mismos tienen una percepción no definida como grupo empresarial a formar joint-ventu re entre empresarios latinoamericano $\left(\mathrm{x}^{2}=1.44\right)$.

El grupo de empresarios Congelados presentan significativamente positiva la percepción a formar joint-venture entre latinoamericanos $\left(x^{2}=21.16\right)$.

En cambio se les observa una tendencia espuria, la percepción a la adquisición de franquicias y otras formas $\left(\mathrm{x}^{2}=-.036\right)$, como también a constituir tradings $\left(\mathrm{x}^{2}=0.36\right)$.

\section{LA INTERDEPENDENCIA ENTRE EMPRESAS PESQUERAS}

Para determinar si los empresarios pesqueros peruanos presentan condiciones intrínsecas para propiciar interdependencias entre sus similares nacionales o del contexto internacional, en vías de conformar relaciones empresariales conjuntas.

Es necesario determinar aquellas variables psicosociales que se correlacionan entre sí; a fin de identificar específicamente que tipo de motivación, percepción e interés faciliten, mantengan y desarrollen la interdependencia empresarial; teniendo en consideración que esta interrelación empresarial es generada, entre otros factores, por la toma de decisiones de los empresarios pesqueros peruanos, las que "direccionarán" su estrategia en el contexto del comercio internacional.

De ahí, que las hipótesis diseñadas se orientan a determinar si hay o no la posibilidad de generar interdependencias en el sector empresarial investigado.

\section{MOTIVACIÓN DE LOGROY AUTORREALIZACION CON INTERESES A LA PESCA SOSTENIBLE}

H1:Los empresarios pesqueros peruanos sí poseen los intereses por el comercio internacional: Pesca Sostenible, Gestión y Mercado del Producto Pesquero que propicien la conformación de relaciones empresariales conjuntas. Esta H1 se cumple parcialmente.

H2: Los empresarios pesqueros peruanos sí poseen las motivaciones: Logro y Autorrealización, e Intereses por la Pesca Sostenible que propicien la conformación de relaciones empresariales conjuntas.

La H2 Rechaza, en razón que no hay relación significativa entre motivación de logro con los intereses de la pesca responsable.

\section{H3:Los empresarios pesqueros peruanos sí}

poseen las motivaciones: Poder y Autorrealización, e Intereses por la Pesca Sostenible que propicien la conformación de relaciones empresariales conjuntas.

Se acepta H3, es decir se dará que los ejecutivos negocien o participen en bloques empresariales por necesidad de poder, en la perspectiva del largo plazo para el desarrollo sostenible.

H4:Los empresarios pesqueros peruanos sí poseen las motivaciones: Logro y Autorrealización, e intereses por la Gestión del Comercio Internacional que propicien la conformación de relaciones empresariales conjuntas. 
Por tanto se llega a deducir por los datos encontrados que la relación requerida en la hipótesis $\mathrm{H} 4$, no se cumple completamente en todos las relaciones, es decir tiene aceptación parcial.

H5: Los empresarios pesqueros peruanos sí poseen las motivaciones: Poder y Autorrealización, e intereses por la Gestión del Comercio Internacional que propicien la conformación de relaciones empresariales conjuntas.

Se rechaza la H5 porque no se cumple la relación significativa con la motivación de poder.

H6: Los empresarios pesqueros peruanos sí poseen las motivaciones: Logro y Autorrealización, e intereses del Mercado del Producto Pesquero que propician la confol7nación de relaciones empresariales conjuntas.

No se identifica correlación alguna, al relacionar los intereses del empresariado hacia la consolidación y ampliación de sus mercados de exportación e interés por la promoción del producto pesquero, con las motivaciones de logro y autorrealización. Por lo tanto, la interrelación exigida en la formulación de la hipótesis H6 no se cumple.

\section{H7: Los empresarios pesqueros peruanos sí}

poseen las motivaciones de Poder y de Autorrealización, e intereses por el Mercado del Producto Pesquero que propicien la conformación de relaciones empresariales conjuntas.

Es notoria la falta de correlación, entre las variables intereses por consolidar o ampliar el mercado del producto pesquero, así como el posicionamiento de la plaza; con las motivaciones de poder y autorrealización. Resultado que posibilita inferir la existencia de lo que podría calificarse como un manejo gerencial de tipo mercantilista, en lugar de aquella denominada gestión empresarial de calidad. Por otro lado hay relación significativa de la motivación de poder y autorrealización con los intereses por el mercado del producto pesquero, por tanto la $\mathrm{H} 7$ cumple parcialmente.

H8:Los empresarios pesqueros peruanos sí poseen percepciones favorables a la modalidad de negocios conjuntos: JointVenture, Trading, Franquicias J' otras que propicien la conformación de relaciones empresariales de negocios conjuntos.

De los resultados presentados sobre la percepción son de tendencia desfavorable en términos generales a las modalidades de negociación conjuntas internacionales; como del análisis especifico por tipo de modalidad se presenta la percepción positiva al Joint Venture, negativa a las Franquicias y espúrea a constituir Training eo. se rechaza la hipótesis H8.

H9:Los empresarios pesqueros peruanos sí poseen percepciones, motivaciones e intereses que propician la conformación de relaciones empresariales conjuntas.

Los empresarios categorizan en base a la significación obtenida por el aprendizaje sobre los negocios empresariales conjuntos, en razón a que la motivación, da direccionalidad o energetiza el comportamiento, es decir ejecutar o tomar las decisiones respectivas; aún cuando las percepciones tienden a ser desfavorables a las franquicias y dubitativas a los tradings.

Lo que nos lleva a indicar que se debe esta condición a una falta de conocimiento o a una distorsión del mensaje sobre estas modalidades empresariales internacionales, como también a la relativa evidencia de éxito experimentada para optar estas formas de estructuración empresarial en el contexto de la cultura nacional e internacional. Igualmente se correlaciona significativamente la percepción a estas modalidades empresariales internacionales con el interés por alcanzar modernidad y calidad de los productos pesqueros por la tecnología. 
Dicha relación nos explica igualmente que a pesar de la tendencia a ser desfavorable la percepción, hay interés por lograr tecnología por intermedio de la estructuración empresarial internacional, la misma es una de las razones fundamentales que trae consigo estas interdependencias empresariales.

Teniendo en cuenta las correlaciones entre la percepción a las modalidades de negocios empresariales compartidos con la motivación. Se identifica que hay una alta interrelación entre ellas con la motivación de autorrealización $(\mathrm{r}=0.56)$ y una adecuada relación por la necesidad de $\operatorname{poder}(\mathrm{r}=0.34)$.

Se desestima que pueda generar interdependencia por la necesidad de logro, porque hay una percepción que pueda dificultar las actividades de la gestión para lograr ascendencia o poder en la dirección por parte de ellos al establecer negocios compartidos por la modalidadjoint-venture, tradings y franquicias u otras modalidades.

Por tanto se acepta parcialmente la hipótesis H9.

\section{CONCLUSIONES Y RECOMENDACIONES}

1. Los empresarios pesqueros peruanos presentan altos niveles porcentuales $(+65 \%)$ de intereses en cada dimensión de las tres áreas diagnosticadas: Pesca Sostenible, Gestión y Mercado del producto Pesquero.

2. Los empresarios pesqueros peruanos presentan niveles promedio de motivación de logro y poder. Estos tipos de motivaciones están favorecidas para el comportamiento gerencial por el alto nivel de la motivación de autorrealización.

3. Existe en el sector una tendencia hacia el desarrollo económico de corto plazo, más no hacia el desarrollo sostenible. En virtud a que los impulsores como es la motivación de logro no presenta altos niveles. Variable que como recurso potencial para orientar, mantener, evaluar y energetizar el comportamiento en posiciones competitivas están mayormente "direccionadas" en el grupo empresarial estudiado por motivos y acciones que puedan diferir paradójicamente para el desarrollo sostenible con la obtención de grandes o máximo beneficios inmediatos.

4. La percepción de los empresarios pesqueros peruanos hacia las modalidades de negocios compartidos: joint-venture, tradings, franquicias entre otras, alcanza una tendencia desfavorable, es decir, la desestiman como posibilidad empresarial. Excepto en el caso de los empresarios de Congelados, estiman favorablemente la modalidad de Joint Venture.

5. Los empresarios pesqueros peruanos presentan una tendencia medianamente favorable hacia la interdependencia lo cual les permitiría insertarse ventajosamente en el mercado global izado.

Sin embargo, sus percepciones hacia los negocios conjuntos internacionales son negativos. Por ello concluimos que la perspectiva del desarrollo sostenido en el corto plazo tiene un pronóstico reservado con tendencia a desfavorable, manteniéndose expectativas positivas por el interés mostrado en las diversas variables descritas para el largo plazo.

6. La interdependencia entre empresas pesqueras peruanas generadas por factores psicosociales hacia la conformación de negocios conjuntos para el comercio internacional se puede constituir en razón a los siguientes resultados.

a) Se identifica correlación significativa entre la motivación de Poder y autorrealización con el interés a la Pesca Sostenible.

b) Se identifica correlación parcial entre las motivaciones de logro y autorrealización 
con los intereses a la gestión del comercio internacional.

e) Se identifica correlación parcial entre los intereses al mercado del producto pesquero con las motivaciones de logro y autorrealización.

d) Se identifica correlación parcial entre los intereses al mercado de productos pesqueros con las motivaciones de poder y autorrealización.

e) Se identifica correlación entre los intereses a la preservación al medio ambiente para el desarrollo del sector pesquero, específicamente, con los intereses a: relaciones empresariales conjuntas, productividad, capacitación del recurso humano del sector pesquero, normatividad institucional de especificaciones técnicas del producto pesquero de exportación.

f) Se identifica correlación de los intereses al aprovechamiento óptimo de los recursos pesqueros para el desarrollo del sector pesquero, específicamente, con: productos pesqueros, desarrollo socio económico de la región y productividad.

7. La generación de interdependencia por las variables psicosociales estudiadas, muestra limitaciones para la conformación de negocios conjuntos entre empresas pesqueras peruanas o con sus similares de la región en razón a los siguientes resultados: (Cuadro $\mathrm{N}^{\circ} 47$ )

a) Se identifica una interrelación no significativa entre las motivaciones de poder y de autorrealización con los intereses a la gestión por el comercio internacional.

b) La percepción tiende a una direccionalidad desfavorable para establecer negocios compartidos: jointventure, tradings y franquicias entre otras modalidades; asimismo no logra correlación con la motivación de poder. e) Se observa que no hay correlación entre la motivación con los intereses a la investigación en el sector pesquero. Asimismo con el interés a la plaza del producto pesquero.

d) Se identifica una correlación entre la promoción del producto pesquero con la gestión de la productividad de la empresa, sin embargo esta relación se presenta negativa.

e) Insuficiente significación de la importancia a la investigación científica y tecnológica, así como al recurso humano no especializado entre las prioridades de los problemas.

f) Se observa en relación a los intereses a la Conservación del recurso pesquero para la pesca sostenible, no se interrelacionan entre si, dificultando así la interrelación de los empresarios pesqueros por este interés.

8. La interrelación significativa de la percepción de los empresarios pesqueros hacia el establecimiento de negocios conjuntos a nivel internacional con el alto interés por modernizar tecnológicamente sus procesos productivos (una de las grandes razones para la alianzas estratégicas en el contexto del comercio internacional) de ahí que, el rechazo a las modalidades de dicha gestión, se puede deber a una disonancia cognitiva entre la información difundida o la que tienen, con la factibilidad de su implementación.

9. Los empresarios pesqueros peruanos tendrían interés por los siguientes propósitos al atribuirle fortaleza y eficiencia a los negocios compartidos por necesidad de motivación de poder y autorrealización:

a) Establecer una red de información comercial oportuna, y a nivel de empresas, detallando los movimientos y transacciones para el mercado internacional.

b) Apoyar la ejecución de negociaciones conjuntas y proyectos de inversión para el desarrollo responsable de la pesca marítima y continental, así como a la acuícola.

e) Concertar acciones por línea de producción manteniendo la libre iniciativa empresarial competitiva.

d) Participación del sector empresarial pesquero para el desarrollo regional en base a la explotación y defensa de los intereses de la producción y comercio de los productos del sector. 
10. Los empresarios opinan que el problema del acceso al financiamiento es uno de los principales del sector, aun cuando el conjunto de las empresas pesqueras tienen un manejo eficiente financiero. La gestión financiera de las grandes y medianas empresas pesqueras, aún cuando el mayor nivel de financiamiento proviene de la Banca destinadas a inversiones de rentabilidad, el financiamiento es proveniente fundamentalmente de proveedores.

11. Lineamientos para el desarrollo de políticas de gestión para la competitividad del sector empresarial pesquero:

a) Fomentar la negociación conjunta e instrumentarla, a fin de lograr competitividad, mediante instituciones gremiales del sector privado y público nacionales $\mathrm{e}$ internacionales

b) Fomentar la necesidad de diversificar, promocionar y publicitar el producto pesquero en el mercado nacional e internacional

e) Desarrollar programas de capacitación en gestión empresarial e implementar programas de motivaciones empresariales, en el contexto interno para el mejoramiento de la productividad.

d) Desarrollar proyectos con programas pilotos para fomentar el desarrollo de pesca responsable y sostenible. así como la utilización de los recursos de alta mar.

e) Identificar y desarrollar habilidades empresariales hacia un mercado globalizado, mediante investigaciones de gestión en el sector y su implementación.

14. La interdependencia empresarial generada por la toma de decisión de los ejecutivos de alta gerencia, estará supeditada a la coincidencia de múltiples factores, entre ellos las variables psicosociales: Interés, motivaciones, percepciones entre otras variables mediadoras para constituir organizaciones tendentes a sustentar la calidad de la gestión; las mismas que deberán continuar con su investigación en la realidad latinoamericana.

15. Como recomendación final del presente trabajo de investigación que brinda a nivel académico la incidencia de las motivaciones, intereses y percepciones del empresariado pesquero nacional en el marco de la economía global izada y altamente competitiva en los mercados internacionales, amerita un análisis con mayor profundidad de carácter concertado entre las instituciones normativas y productivas.

\section{BIBLIOGRAFÍA}

Cárdenas, G.(1997). Economía Nacional e Internacional. Serie Lecturas I, II, III y IV. Lima. UNMSM.

Davis, K. \& Newstrom, J. (1994). El Comportamiento Humano en el Trabajo. Comportamiento organizacional. México DF. MX Graw Hill. Octava Edición.

Drucker, P. (1993). La Sociedad Poscapitalista. Barcelona Ed. Apóstrofe. SI. Primera Ed. FAO/CCRF/TC 1994, Informe Consulta Técnica sobre el Código de 1994 Conducta para la Pesca Responsable. Roma. Sl. Edit.

Hall, Richard H. 1983, Organizaciones, Estructura y Proceso. México DF. Ed. Prentice may Hispanoamericana. Reimpresión Español.

Hodgetts, Richard \& Altman, Steven, 1989, Comportamiento en las Organizaciones México DFMc.GRAW-HILUInteramericana.SA 1era Ed. Español.

Instituto Peruano de Administración de Empresas- IPAE, 1994, X Congreso Nacional de Gerencia. Lima.

Instituto Peruano de Administración de Empresas- IPAE. 1994, Anchoveta Peruana: una alternativa alimenticia para el futuro. Lima. Edit. ITP

INFOPESCA/FAO, 1992, Directorio de Exportaciones de Productos Pesqueros de América Latina y El Caribe. S/edit. 
Katona, George, 1965, Análisis Psicológico del Comportamiento. Madrid. RIALP S.A. 1 era. Edic. Español.

Kerlinger, Fred, 1988,Investigación del Comportamiento. México DF- Mc. Graw Hill. Tercera Edic. Español.

Kolb, David A. y Colaboradores, 1977, Psicología de las Organizaciones. México DF Prenti1ce-HALL Hispanoamericana S.A. 1 era. Ed. Español.

Kotter, John P. \& Heskett, James, 1992, Corporate Cultural and Performance. NY, USA The Free Press.

Leavitt, Harold J. \& Bahram, Horna, 1988, Managerial Psychology, Managing Behaviour in Organizations. The University of Chicago Press. Fifth Edition.

Luthans, Fred \& Kreitner, Robert 1988, Modificación de la conducta Organizacional. México DF. Edi. Trillas. Sexta Reimpresión.

Mc Clelland, David C. \& Winter, David G., 1970, Cómo se motiva el éxito económico. México DF. UTEHA. 1 era. Ed. Español.

Machado, Fernando, 1994, La política Industrial y la Política Tecnológica. En: Los Retos Actuales. Capitulo $\mathrm{N}^{\circ}$ 48.Caracas. Comité Editorial Capítulos del SELA.

Wadsen. K.B. 1965, Teorías de la Motivación. Un estudio comparativo de las teorias modernas de la Motivación. Buenos Aires. PAIDOS.

Michel, John G. \& Hambrick, Donald, 1992, Diversificación Posture and Top Management Team Characteristics. Mew York. Academy of ManagementJoumal. Vol. 35. $\mathrm{N}^{\circ} 1$.

Mintzbeerg. Henry \& Quinn, James Brian, 1988, El Proceso Estratégico: Conceptos, contexto y casos. México DF. Prentice-HALL Hispanoamericana. Segunda Edición en Español.

Montecinos, Gladys y Colaboradores, 1986, Psicología Social. Biblioteca de Psicología Peruana.

Moreno, Ornar, 1988, Hacia un Nuevo Enfoque de las Relaciones del Trabajo y el Papel del sindicalismo En: Nuevo Paradigma Productivo en América Latina. Buenos Aires. Editorial Asociación Trabajo y Sociedad.

Nasser, Martin E. \& Vivier, Frank J., 1993, Mindsetfor the New Generation Organization. Republic of South Africa. Published JUTA \& Co. Ltd. First Published.

Novak Talavera, Fabián, 1995, Agenda Internacional. Instituto de Estudios Internacionales. Año II $\mathrm{N}^{\circ}$ 5. Lima PUCO

OLDEPESCA, 1995, Informe a la XI Conferencia de Ministros de OLDEPESCA. Caracas. Agosto 1995. Lima S/edit.

OLDEPESCA, 1996, Informe Estadístico Pesquero 1995. Lima S/edit.

OLDEPESCA, 1997, Situación Regional de Pesca y Acuicultura 1996. Lima S/edit.

ONU/FAO, 1992, La ordenación y Planificación Pesquera y la Reactivación del Sector Pesquero en el Perú GCO/1NT/466/NOR. Información de Campo. Roma.

Osgood, Charles, 1974, Curso Superior de Psicología Experimental. Método y Teoría. México DE TRILLAS $3^{\text {ra }}$ Reimpresión Español.

Paredes Tarazona, Mildred, 1997. La Interdependencia Empresarial Factor de Calídad en la Gestión-Caso Sector Empresarial Pesquero Peruano. Tesis para optar el Grado de Magíster. UNMSM. Escuela DE Post Grado- FCCAA

PESCA, 1978, Revista de Circulación Mundial Colección 1978 Enero-Diciembre. S/edit.

Pierce, Jon L., 1993,Manager's Bookshelf. A. Mosaic of Contemporary J'iews. New York. Harper Collins Collage Publishers. Inc. Third Edition.

PROGRAMA DE LAS NACIONES UNIDAS PARA EL DESARROLLO - PNUD., 1996, Informe Sobre Desarrollo Humano. Lima Ed. Mundi-Prensa. 
Porter, Michael, 1995, Estrategia Competitiva. Técnicas para el Análisis de los Servicios Industriales y de la Competencia. México DE

Rivera Benavides, Ángel, 1997, Historia del Medio Ambiellte en la Agencia Internacional Boletín Año 2 "El Economista" UNAFV -FCCE. LimaS/edit.

Robbins, Stephen, 1994, Comportamiento Organizacional. Conceptos controversias y Aplicaciones. México DF Prentice-HALL Hispanoamericana. Sexta Ed.

Santoro, Eduardo y Colaboradores. 1980, Psicología Social. México DF Trillas. Primera Reimpresión.

Serapio, Manuel \& Cascio, Wayne E, 1996,EndCames in International Alliances. New York

Vol. X N ${ }^{\circ} 1$ February. The Academy of Management. Executive.

Sherif, Muzafer \& Sherif, Carolyn w., 1974, Psicología Social. México DE HARLA S.A. 3era.Ed.

Strumpel, Burkhard y Colaboradores., 1979, La Conducta Humana en las Relaciones Económicas. México DF Trillas primera Edición en Español.

Toffler, Alvin, 1995. Shock del Futuro. Barcelona, Plaza \& Janes Editores S.A. Novena Vicuña Peri, Luis, 1991, Manual de Inventario de Intereses Profesionales y Ocupaciones. Lima CASM-84-R-91.

Wolman, Benjamin B. 1980. Manual de Psicología General. Barcelona Martínez Roca S.A. 


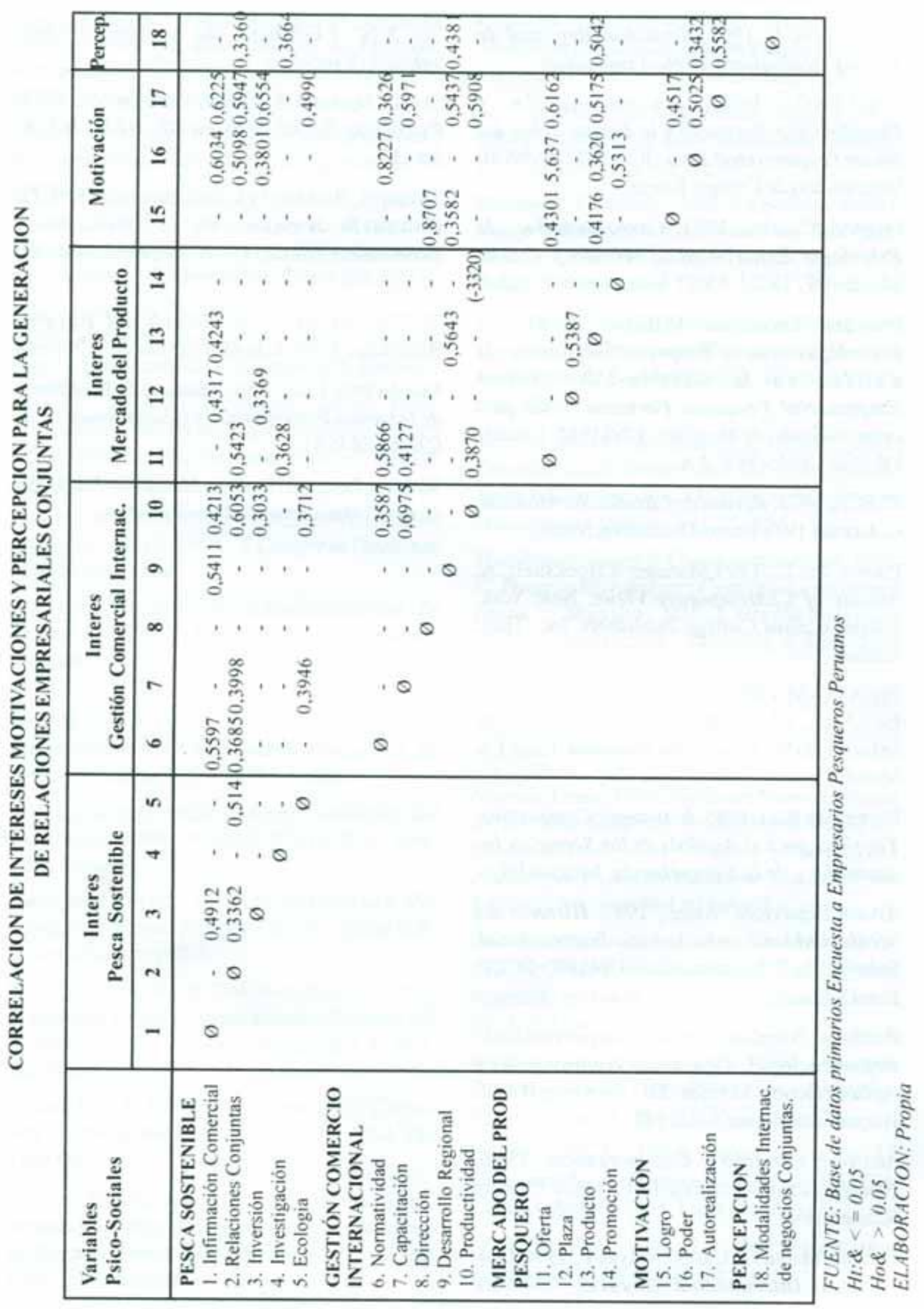

